\title{
Les consultants et la réforme des services publics
}

La Révision générale des politiques publiques (RGPP) est lancée le 7 juillet 2008. Après un processus de réorganisations administratives démarré en 1992, processus surdéterminé par les jeux complexes de concurrence et d'alliance entre décideurs politiques, grands corps de l'État, élus locaux, syndicats de fonctionnaires, la « réforme de l'État » territorial, qui voit fusionner les services déconcentrés de l'État en huit grandes directions régionales, semble, avec la RGPP, passer en mode industriel. Déjà, en 2001 , le vote de Loi organique relative aux lois de finances (LOLF) avait bousculé le subtil équilibre politique entre le ministère de l'Intérieur, le ministère de la Fonction publique et le ministère des Finances; ce dernier s'est alors retrouvé à la tête de lignes managériales très centralisées, « pilotant » l'activité des ministères à l'aide d'indicateurs de performance, avec comme impératif catégorique la rationalisation des dépenses publiques. Dans le prolongement de cette dynamique de « budgétisation » de la réforme de l'État, le ministère des Finances s'est doté, en 2005, d'une nouvelle direction générale de la modernisation de l'État (DGME). Peu de temps après, en 2006-2007, des « audits de modernisation » sont lancés pour passer au crible les politiques publiques afin d'identifier les sources potentielles d'économies budgétaires.

\section{La RGPP : Centralisation, politisation et technocratisation de la « réforme de I’État »}

Dans le contexte de politisation de la question de la dette publique et de l'offensive des think tanks néolibéraux, l'élection de Nicolas Sarkozy à la présidence de la République et la nomination à des postes gouvernementaux clés de personnalités souvent issues, non de la haute fonction publique, mais du monde des affaires, systématisent et radicalisent les évolutions amorcées au début des années 2000. Un conseil de modernisation des politiques publiques (CMPP), placé sous la présidence du président de la République, est mis sur pied, tandis qu'est enclenchée l'ambitieuse Révision générale des politiques publiques (RGPP), inspirée de l'expérience canadienne (Canadian Program Review). Le dispositif est à la fois souple, très centralisé, très technocratique et très opaque puisqu'il associe, au sein de petites task forces, les plus hauts niveaux politiques (I'Élysée et Matignon), des membres des corps d'inspection ministériels et interministériels et, surtout, des consultants issus des grands cabinets internationaux. Les objectifs sont clairs pour les ministères ainsi « évalués » : il leur faut impérativement réduire leurs dépenses, repenser leur rôle et leurs missions, éventuellement en favorisant l'externalisation vers le privé de la mise en œuvre des politiques publiques dont ils ont la charge. Pas moins de 332 décisions de réorganisation sont ainsi arrêtées pour la période 2009-2011.

Pourtant, à compter de 2010, plusieurs rapports publics critiquent vertement à la fois les modalités et les résultats de la réforme de l'État version RGPP. Ainsi, le Conseil économique, social et environnemental exige de «suspendre

temporairement, pour évaluer et réorienter, la Révision générale des politiques publiques » car, selon l'auteure de ce rapport : «Au terme de quatre années de restructurations, la RGPP suscite des critiques convergentes (commissions parlementaires, Cour des comptes, corps de l'inspection générale, ensemble des associations d'élus, professionnels exerçant dans les administrations publiques, organisations syndicales de salariés, plusieurs associations, etc.). Ce fait, assez exceptionnel pour être souligné, justifie la suspension de la RGPP afin d'évaluer ses effets avant d'opérer les réorientations qui en découleront. En effet, les griefs exprimés sont aussi assortis de recommandations précises portant sur l'essence de la réforme, les méthodes de sa conduite, les procédures de suivi et d'évaluation. [...] À cet égard, des critiques soulignent l'absence de recherche de l'implication des citoyens ("le citoyen est souvent le grand oublié") dans une réforme qui les concerne pourtant. »

L'on retrouve des critiques proches dans le rapport du médiateur [voir encadré « Les services publics : fusion ou fission ? », ci-contre], qui dénonce de son côté des « politiques publiques brouillées par l'empilement législatif », «les dommages collatéraux dus aux réformes précipitées », « des ambitions politiques qui se heurtent aux moyens limités », «la méconnaissance des textes par les citoyens », « une loi pas toujours applicable ». L'on pourrait étendre aisément ce diagnostic à l'ensemble des fusions administratives de l'État territorial (comme les agences régionales de santé ou les préfectures). Toujours en 2010, une commission sénatoriale mise en place à l'initiative du groupe socialiste, estime que la RGPP « n'a fait l'objet d'aucune évaluation de ses effets, en dehors des rapports d'étape soumis au conseil des ministres [...]. Au Parlement, aucun débat public n'a eu lieu. Les effets de la RGPP ne sont étudiés qu'au travers des rapports budgétaires qui, au Sénat, sont très critiques ». Ici, c'est le processus décisionnel centralisé et technocratique de la RGPP qui est mis en cause. Ces trois rapports publics ont en commun d'être le fait de catégories d'agents évincés des décisions de réorganisation administrative : «corps intermédiaires » (parlementaires, syndicats) dans le cas du Conseil économique, social et environnemental et du Sénat, « usagers » dans le cas du rapport du Médiateur de la République.

Cela étant, les critiques fusent aussi du côté des institutions qui devraient, au contraire, se reconnaître dans la démarche essentiellement budgétaire de la RGPP. Ainsi, la Cour des comptes remet en question les économies réalisées ou prévues par la RGPP, soit sept milliards pour la période 2009-2011 et dix milliards pour la période 2011-2013. En 2010, elle questionne « la traçabilité des décisions mises en œuvre, notamment de leurs effets budgétaires, insuffisante. Leurs évolutions ne sont jamais présentées dans les rapports successivement publiés et la RGPP se présente comme un flux de décisions permanentes, alors que leur contenu évolue avec le temps ». 100000 postes de fonctionnaires sont, en effet, supprimés entre 2007 et 2009. Cependant, ces suppressions ne semblent pas avoir engendré d'économies budgétaires substantielles car, d'une part, la moitié des gains de l'application du principe de non remplacement d'un fonctionnaire sur deux a été rétrocédée à certains fonctionnaires et, d'autre part, beaucoup de ministères ont surestimé le nombre de fonctionnaires partant en retraite. Au final, des trois objectifs assignés à la RGPP - économies budgétaires, « amélioration de la qualité du service rendu aux usagers », « modernisation des administrations publiques »-, aucun ne semble avoir été atteint. Le constat de la multiplication de décisions prises «en chambre », de mises en œuvre précipitées et autoritaires, de systèmes de décision de 
plus en plus verticalisés, de l'absence d'implication des agents de la fonction publique et des « usagers », du défaut d'évaluation des effets des mesures décidées, de l'opacité des conséquences budgétaires n'empêche pas, dans le contexte de dramatisation de l'état des finances publiques, la RGPP d'être reconduite, sinon intensifiée.

\section{La double réalité de la réforme de l'État version RGPP}

La RGPP est emblématique de la double réalité de la « réforme de l'État » et des services publics : d'un côté, le périmètre d'intervention directe de l'État, selon les modalités caractéristiques de l'après-guerre (nationalisations, services publics, développement du fonctionnariat, etc.), est réduit, de l'autre l'action publique connaît une « caporalisation » concomitante de l'acclimatation à la « nouvelle gestion publique » dans la haute administration française. Cette double réalité met à mal la thèse, longtemps en vogue dans les années 1980 et 1990, y compris - et surtout - dans les sciences sociales, de l'« évanescence de l'État», du « retrait de l'État», de l'avènement de la « gouvernance dépolitisée » et de l'« État creux ». Emblématique de cette thèse est l'ouvrage de Susan Strange, spécialiste de relations internationales. Constatant la montée en puissance de nouvelles catégories d'agents - assureurs et opérateurs de la finance de marché, cabinets de conseil, mafias, « éconocrates » des organisations internationales, multinationales -, celle-ci soutient que « les forces impersonnelles des marchés mondiaux - davantage organisées depuis l'après guerre, par l'entreprise privée via la finance, l'industrie et le commerce que par les décisions conjointes des gouvernements - sont désormais plus puissantes que les États censés incarner l'autorité politique ultime sur la société et l'économie. »

En fait, il semble que l'« évanouissement » de l'État était davantage dans l'œil des sociologues et des politistes, poussés aux audaces conceptuelles par la concurrence académique et par la dépendance à la demande sociale, que dans la réalité étatique elle-même. En particulier, la « dé-liaison » des recherches sur l'État, sur l'administration et sur l'action publique, au profit de ces dernières, a conduit les chercheurs français à insister excessivement sur les phénomènes de fragmentation, sinon de dispersion, et d'ouverture, de l'État. De fait, avec le regain d'intérêt, à partir de la fin des années 1990, pour l'État, les structures administratives et le travail des fonctionnaires, petits et grands, le balancier repart dans l'autre sens : les réflexions récentes sur la rationalité politique néolibérale, ou encore les recherches empiriques sur les réformes des services publics inspirées du New Public Management, rappellent aux chercheurs en sciences sociales combien les agents étatiques continuent de jouer un rôle-clé dans les dynamiques de recomposition des États. Dans certains cas, comme en Grande-Bretagne mais aussi en France, les réformes, loin de déboucher sur le retrait de l'État, participent d'une recentralisation et même d'une « seconde révolution bureaucratique ».

Aussi, on ne peut s'étonner que, confrontés à une certaine permanence de l'État, certains sociologues ou politistes en appellent à la définition de nouveaux concepts aptes à saisir aussi bien les recompositions que les permanences de la réalité étatique. En cela, ils rejoignent les réflexions d'économistes hétérodoxes, tel James K. Galbraith qui définit l'État contemporain comme un «État prédateur »: «Une coalition d'adversaires implacables du cadre réglementé dont dépend l'intérêt public, composée d'entreprises dont les principales activités lucratives concurrencent en tout ou partie les grands services publics de l'increvable New Deal. Une coalition qui cherche à prendre le contrôle de l'État, pour empêcher l'intérêt public de s'affirmer mais aussi pour braconner dans les flux économiques créés par l'intérêt public passé. [...] Aucune de ces entreprises n'a intérêt à rétrécir l'État, et c'est ce qui les distingue des conservateurs à principes. Sans l'État et ses interventions économiques, elles n'existeraient pas elles-mêmes, et elles ne pourraient pas jouir du pouvoir de marché qu'elles sont parvenues à exercer. Leur raison d'être est plutôt de tirer de l'argent de l'État - tant qu'elles le contrôlent. » En effet, l'État néolibéral n'intervient pas moins que l'État d'après-guerre, il intervient selon d'autres voies et d'autres finalités : introduction d'instruments visant à instiller de la concurrence dans la sphère publique, « régulation » de la concurrence entre « opérateurs » privés et publics dans la fourniture de certains services publics, mise au point de batterie d'indicateurs de mesure de la « performance », encadrement des conduites individuelles par des systèmes d'incitation informationnelles et monétaires, etc.

\section{Au-delà de l'alternative public/privé : repenser l'État ?}

Autrement dit, le couple notionnel État/marché, si profondément ancré dans les têtes, est un obstacle épistémologique à la saisie de la spécificité de l'État néolibéral et des logiques de prédation de la sphère publique qu'il soutient et alimente. La privatisation et la libéralisation ne sont en aucune façon le résultat de pressions externes de nature économique (« la globalisation ») mais relèvent bien d'un projet politique défendu par certaines élites étatiques alliées à des intérêts privés, comme les cabinets de conseil. II en résulte non une « désétatisation » de la société et un recul de la bureaucratie, mais au contraire la poursuite de l'étatisation de la société par d'autres moyens, au service d'intérêts différents que ceux que l'État servaient dans la période 1945-1980, et l'avènement de nouvelles formes bureaucratiques que l'on pourrait qualifier de « néolibérales ». Cette bureaucratie revue et corrigée par le New Public Management, diffère de la bureaucratie wébérienne classique en ce qu'elle apparaît plus fragmentée (en «agences ») et gouvernée par le principe de concurrence, en ce qu'elle est équipée de nouveaux instruments d'action publique comme la contractualisation et le pilotage par les indicateurs, en ce qu'elle promeut une posture consumériste là où les formes antérieures s'adressaient à des « assujettis » ou des « citoyens ». A posteriori, ces inflexions de la recherche académique valident la posture théorique et méthodologique de Pierre Bourdieu. Refusant le point de vue scolastique des travaux d'inspiration marxiste et fonctionnaliste, plaidant pour des analyses de longue période ancrées empiriquement, le «structuralisme génétique » de Pierre Bourdieu, et à sa suite, les travaux de 


\author{
http://www.arss.fr/articles/les-consultants-et-la-reforme-des-services-publics/
}

socio-histoire sont logiquement conduit à mettre l'accent sur la dynamique de long terme d'étatisation et de « mise en administration » de la société. L'histoire structurale de l'État que Pierre Bourdieu appelait de ses vœux permet d'éviter de tomber dans le piège de la « nouveauté radicale » et de céder aux sirènes des discours réformateurs pour mieux replacer les changements observés dans le court et moyen terme de l'histoire longue des trajectoires étatiques nationales. Ce faisant, la sociologie rejoint les conclusions des nombreux travaux anglo-saxons d'inspiration néo-institutionnaliste, à dominante sociologique ou historique, qui montrent comment l'acclimatation du New Public Management se fait sous contraintes institutionnelles nationales, selon des voies parfois inattendues et des résultats qui ne le sont pas moins.

\title{
Pour une histoire structurale de l'État et de sa « réforme »
}

II s'agit donc de tenir la dialectique des projets et stratégies réformateurs (le court et moyen terme) d'un côté, des institutions (le long terme) de l'autre. Cette entreprise va de pair avec la volonté d'identifier précisément les intérêts mobilisés autour des « réformes » à rebours des analyses qui font appel à des processus abstraits pour expliquer (voire légitimer) les changements observés ou des dénonciations générales invoquant des intérêts très généraux (le «patronat », les « multinationales », etc.). Ainsi devient-il possible de combler un vide préoccupant, ainsi que le notait récemment Ezra Suleiman : « en d'autres termes, la réforme de l'administration publique, comme toutes les réformes, a ses gagnants et ses perdants. On n'a encore entrepris aucune analyse pour chercher à savoir qui pourraient être les principaux bénéficiaires de ces réformes », propos qui font étrangement écho à ceux, antérieurs de vingt ans, de Susan Strange : « Se plonger dans les détails pratiques d'une technique, ou dans les processus de décision stratégique des entreprises ou des ministères, est une bonne façon de mettre à l'épreuve les abstractions de la théorie, quitte à modifier celle-ci ou à élaborer des schémas d'explication différents. » Il est donc urgent pour les sciences sociales de s'atteler à cette tâche de caractérisation sociologique des coalitions qui définissent et promeuvent les réformes et les intérêts que celles-ci servent, mais toujours sous contraintes institutionnelles et politiques. La RGPP constitue ainsi, de ce point de vue, un moment ou, mieux, une configuration spécifique des agents mobilisés autour de la « réforme de l'État », configuration dont les spécificités ne peuvent être appréciées qu'au regard des configurations antérieures du « souci de soi de l'État » : l'association des plus hauts niveaux politiques, des grands corps d'inspection et de grands cabinets de conseil dessine une coalition très différente de celles observées par le passé. Cependant, parce qu'elles ont défini peu à peu une catégorie et un secteur d'action publique spécifique où l'État se prend lui-même comme objet de réforme, les séquences précédentes ont rendu possible, sans toutefois la rendre inéluctable, l'avènement de cette « rupture » qu'est la RGPP.

Le dossier ici présenté met en évidence la recomposition de l'économie des échanges entre les élites (politiques, administratives et économiques) constitutives du champ du pouvoir et la « fluidification » de la circulation entre ces trois espaces sociaux. Dans cette perspective, la RGPP oppose moins secteurs public et privé qu'élites d'un côté, fonctionnaires subordonnés et « usagers » de l'autre. En effet, loin de se conformer aux signes, vocabulaire et pratiques de la «modernité » managériale, soi-disant spécifiques au secteur privé, une fraction de l'élite de l'État, progressivement convertie aux finalités néo-managériales, produit savoirs et pratiques ad hoc et s'appuie, pour la mise en œuvre des réformes et le contournement des résistances qu'elles soulèvent, sur le savoir-faire des grands cabinets (notamment américains) de conseil. Si le poids de ces derniers se trouve renforcé au sein de l'État, les experts sont cependant maintenus dans une position subordonnée vis-à-vis des élites administrative et politique. Cette position modeste et technique des experts est même la condition de leur intégration au sein des dispositifs institutionnels de prise de décision, comme le montre l'analyse diachronique du processus (très discret) de privatisation de l'assurance maladie.

Sans préjuger de son caractère inéluctable ou irréversible, la reconfiguration des rapports de forces qui structurent aujourd'hui l'État apparaît comme une des clés explicatives de la montée en puissance d'une telle expertise gestionnaire. Le développement, plus tardif en France qu'en Grande-Bretagne ou au Canada, d'une industrie du conseil en réforme de l'État, est moins lié à l'affaiblissement des grands corps de l'État (et tout particulièrement des corps d'ingénieurs), et de leurs institutions de reproduction, qu'à leurs profondes mutations. Adopter une telle perspective nécessite de rompre avec une lecture internaliste de l'État et ouvre de nouvelles pistes de recherche replaçant les temps forts des réformes des services publics dans les transformations structurales de plusieurs sous-champs du champ du pouvoir : le champ politique, avec la technocratisation des principaux postes à responsabilité ; le champ bureaucratique, avec la montée en puissance, perceptible depuis l'après-guerre, d'un nouvel ethos, fondé sur l'expertise économique et comptable, au détriment du droit, la politisation plus récente de la haute fonction publique, le désintérêt de l'élite des hauts fonctionnaires pour les postes publics au profit du pantouflage dans le secteur privé, considéré comme plus à même de « booster » une carrière, puis leur retour au sein de l'État ou encore la « gestionnarisation » d'administrations qui avaient construit leur identité contre celle de l'administration des Finances) ; le champ des Grandes écoles (la montée en puissance du modèle des business schools et l'internationalisation des institutions de formation des serviteurs de l'État).

Ces transformations de la structure de l'État sont à mettre en relation avec les changements morphologiques des élites économiques, et plus globalement avec la montée en puissance, au sein du champ du pouvoir, des élites issues du commerce, de la finance et du conseil (au détriment par exemple des élites industrielles). Elles sont également à rapporter à l'internationalisation et à la concentration de l'espace du conseil. Entamé au cours des années 1980, lorsque les ingénieurs issus des grandes écoles nationales, occupant jusque-là les positions dominantes de cet espace, se voient contraints d'acquérir une formation à la gestion - à I'INSEAD, HEC ou au sein d'une business school américaine - pour conserver leur emprise sur les cabinets les plus convoités, le processus de restructuration de l'espace, au profit d'un pôle économique incarné par les grands cabinets anglo-saxons et américains, s'accélère avec la nécessité de développer des prestations de haute technicité (telles que les Enterprise Resource Planning, ERP) permettant de restaurer la croyance dans une profession marquée alors par les scandales financiers. Les mutations observées au sein de l'espace du conseil ont largement précédé celles qui s'affirment depuis une dizaine d'années au sein des institutions de formation et de recrutement des élites 
administratives, comme si l'univers du conseil formait, lors des années 1990, une sorte de prototype social, un laboratoire au sein duquel sont collectivement encouragées les dispositions constitutives d'un « néo-sujet » : brouillage des identités sociales, contestation des hiérarchies scolaires établies, dépassement de soi, surinvestissement dans le travail considéré comme support privilégié de réalisation personnelle, exacerbation de l'esprit de concurrence.

\section{Ce que font les cabinets de conseil à l'État}

Ces transformations conjointes expliquent en dernier ressort que, bien que la réforme de l'État ait constitué depuis plus d'un siècle un objet de convoitise pour les cabinets de conseil, les consultants n'aient été que récemment associés au plus haut niveau de sa mise en œuvre. L'analyse diachronique des relations établies entre certaines administrations et les consultants fait apparaître, pour chaque contexte, les conditions de leur réception au sein de l'État, liées, au-delà des alliances politiques, à la force des arrangements institutionnels hérités de l'histoire : dé-liaison, avant la Seconde Guerre mondiale, entre la critique libérale et la critique gestionnaire de l'État, prise en charge de réformes par « petites touches » de l'État par des ingénieurs issus des grands corps et favorables à un interventionnisme établissant les conditions d'une concurrence généralisée ; renoncement, en l'absence d'une politique publique, à l'élaboration d'une doctrine générale à vocation théorique (le management public) et balkanisation, des années 1970 au début des années 1990, des savoirs gestionnaires portés par les cabinets de conseil. Lorsque le compromis historique est remis en cause, au cours des années 1990, c'est à l'initiative de certains hauts fonctionnaires, producteurs d'un savoir global et porteurs d'une politique publique, les consultants, voués jusque-là aux interventions techniques et parcellisées, ne disposant pas alors d'une véritable assise institutionnelle au sein de l'État. Si la position subordonnée des cabinets de conseil (et des experts extérieurs à l'État) semble constituer un invariant historique des processus de réforme, reste alors à déterminer ce que les consultants font réellement, aujourd'hui, à l'État.

D'abord, ils équipent techniquement les hauts fonctionnaires chargés du pilotage des commissions dédiées à l'élaboration de la nouvelle architecture de l'État (et tout particulièrement au sein de la Direction générale de la modernisation de l'État, DGME), offrant des prestations de management des systèmes informatiques ou encore de Lean management. De surcroît, ils participent à une véritable «mise sous perfusion » idéologique : important (traduisant, adaptant, diffusant, etc.) idées et standards de gestion, « ouvrant les esprits » et convertissant les clients potentiels aux concepts qu'ils agitent. Endossant, au sein des think tanks consacrés à la modernisation de l'État, le rôle de « passeur » entre élites administratives, politiques et économiques, ils rendent plus perméables les frontières entre ces groupes et jouent de leur position « d'agents doubles » pour faire converger les intérêts et développer les liens entre ces élites, donc favoriser leur intégration.

Ensuite, ils disposent d'un véritable savoir-faire dans l'art d'accompagner les réformes, de « communiquer » et de vaincre les résistances. Au sein d'EDF, par exemple, ils s'appuient sur certaines dispositions sociales - comme la croyance dans l'excellence technique - d'agents occupant des positions dominées qu'ils promeuvent alors responsables du projet de « modernisation ». Dans d'autres services publics, comme l'audiovisuel, ils adoptent une novlangue gestionnaire, souvent incompréhensible pour les salariés, mais dont l'efficacité symbolique, on pourrait presque dire « magique » tant elle paralyse, repose en partie sur l'intériorisation de la disqualification de la bureaucratie d'État et de ses fonctionnaires - i.e. de leurs « privilèges » et de leurs « archaïsmes »--, intériorisation qui est elle-même le produit d'un très long processus d'inculcation. Enfin, et c'est sans doute l'un des effets les plus paradoxaux de l'imposition d'un management néolibéral porté par des directions souvent issues du monde du conseil, la réforme des services publics engendre un recours accru, dans les entreprises publiques récemment transformées en sociétés anonymes, aux cabinets de conseil. Lorsqu'il ne suscite pas des stratégies de retrait, ce nouveau management peut favoriser l'intervention, à la demande des Institutions représentatives du personnel (IRP), de cabinets de contre-expertise agréés par le ministère du Travail. Si la possibilité d'un recours à ce type d'expertise est ancienne, le secteur est aujourd'hui en plein essor, la fermeture des élites sur elles-mêmes favorisant le développement d'autres formes de résistance collective. Cependant, comme le montre le cas de France Télécom Orange, le recours à une telle expertise ne fait pas l'unanimité au sein de l'univers syndical : outre le fait qu'elle tend à se substituer à l'expertise à laquelle prétendent traditionnellement les syndicats (expertise fondée sur des savoirs juridiques) et à importer dans cet univers les règles et les normes du champ du conseil, auquel participent ces cabinets atypiques, une telle expertise contribue à transformer profondément le registre de l'action syndicale et engendre des effets spécifiques qui restent à analyser.

Pour citer l'article.

Henry, Odile ; Pierru, Frédéric (2012, juin). "Les consultants et la réforme des services publics". Actes de la recherche en sciences sociales, $n^{\circ} 193$, p.4-16 